\title{
Characterization of Patients With Refractory or Unexplained Chronic Cough Participating in a Phase 2 Clinical Trial of the P2X3-Receptor Antagonist Gefapixant
}

\author{
Alyn H. Morice ${ }^{1}$ D . Surinder S. Birring ${ }^{2}$ - Jaclyn A. Smith ${ }^{3}$. Lorcan P. McGarvey ${ }^{4}$ Jonathan Schelfhout ${ }^{5}$. \\ Allison Martin Nguyen ${ }^{5} \cdot$ Zhi Jin Xu ${ }^{5} \cdot$ Wen-Chi Wu ${ }^{5} \cdot$ David R. Muccino ${ }^{5} \cdot$ Mandel R. Sher $^{6}$
}

Received: 25 January 2021 / Accepted: 6 March 2021 / Published online: 7 April 2021

(c) The Author(s) 2021

\begin{abstract}
Purpose This analysis assesses clinical characteristics of patients with refractory chronic cough (RCC) or unexplained chronic cough (UCC) enrolled in a phase 2 study to better understand this patient population.

Methods Patients with RCC/UCC lasting for $\geq 1$ year and cough severity visual analog scale (VAS) score of $>40 \mathrm{~mm}$ at screening were eligible. Demographics, clinical characteristics, and medical history were collected at baseline. Coughrelated measures included cough severity VAS, Cough Severity Diary (CSD), Leicester Cough Questionnaire (LCQ), and a structured cough-trigger questionnaire. Medication history included all medications 30 days before screening and chronic cough treatments within 1 year before screening. Data were summarized using descriptive statistics.

Results Patients ( $N=253$; female, 76\%; mean age, 60 years) had severe (mean cough severity VAS, $57.5 \mathrm{~mm}$ ) and longlasting (median duration, 11 years) cough. The most burdensome self-reported aspects included psychological and social factors (LCQ) and cough frequency and intensity (CSD). Patient-reported triggers were consistent with cough hypersensitivity (e.g., $95 \%$ to $96 \%$ reported irritation or tickle in throat). Common reported comorbidities included gastroesophageal reflux disease (GERD; 56\%), allergic rhinitis (47\%), and asthma (30\%); 12\% of patients had been diagnosed with all 3 conditions. The most common prior medications included inhaled or oral steroids (21\%), antihistamines (15\%), and antacids (15\%). Conclusion Patients with RCC/UCC had severe, long-lasting, and burdensome cough with clinical features of cough hypersensitivity. Many patients had been diagnosed with GERD, allergic rhinitis, and asthma but had a persistent cough despite treatment of these conditions.
\end{abstract}

Trial registration: ClinicalTrials.gov, NCT02612610; registered November 20, 2015

Keywords Persistent cough $\cdot$ Troublesome cough $\cdot$ Refractory cough $\cdot$ Idiopathic chronic cough $\cdot$ Cough hypersensitivity syndrome

Alyn H. Morice

a.h.morice@hull.ac.uk

1 Respiratory Research Group, Hull York Medical School, Cottingham, UK

2 Centre for Human \& Applied Physiological Sciences, School of Basic \& Medical Biosciences, Faculty of Life Sciences \& Medicine, King's College London, London, UK

3 Division of Infection, Immunity, and Respiratory Medicine, University of Manchester, and Manchester University NHS Foundation Trust, Manchester, UK

4 Wellcome-Wolfson Institute for Experimental Medicine, Queen's University Belfast, Belfast, UK

5 Merck \& Co., Inc., Kenilworth, NJ, USA

6 Center for Cough, Largo, FL, USA

\section{Introduction}

Chronic cough (CC), defined as a cough lasting $>8$ weeks, has a global prevalence of $\sim 10 \%[1,2]$. Patients with CC can experience physical burden as a result of their cough and negative effects on their social lives and psychological wellbeing [3-5]. Chronic cough is often long lasting, persisting for several years and sometimes decades [5-8]. There is poor recognition that $\mathrm{CC}$ can be refractory to treatment of associated conditions or unexplainable; thus, patients with $\mathrm{CC}$ are frequently labeled as having other conditions, and such confusion often leads to multiple diagnoses being suspected $[9,10]$. Diagnostic uncertainty may lead to repetitive medical consultations, fruitless investigations, and unnecessary 
treatment trials $[4,11,12]$. Patient burden is exacerbated by the current paucity of effective and safe treatment options for these patients, with no available pharmacologic treatments having approved indications for $\mathrm{CC}$.

Intervention fidelity, defined as the "extent to which an intervention was delivered as conceived and planned to arrive at valid conclusions concerning its effectiveness in achieving the target outcomes," is important for reliably identifying or excluding potential conditions associated with CC [13-15]. Diagnostic workup for CC includes the following steps: assessment of medical history to address factors that could contribute to cough (e.g., smoking; use of drugs that elicit cough, such as angiotensin-converting enzyme [ACE] inhibitors; environmental exposures); imaging and clinical assessments to identify red flags suggestive of lifethreatening conditions; and differential diagnosis of potential comorbid conditions $[1,10]$. Several medical conditions can be associated with $\mathrm{CC}$, including gastroesophageal reflux disease (GERD), asthma, nonasthmatic eosinophilic bronchitis, and upper-airway cough syndrome (UACS). However, many patients with these conditions do not have CC, suggesting $\mathrm{CC}$ may be driven by distinct mechanisms [10]. Indeed, many patients with CC continue to cough despite optimal assessment and treatment of presumed associated conditions; these patients are often referred to as having refractory CC (RCC) $[1,10,13]$. Additionally, CC can be present in patients who do not have identifiable or treatable conditions associated with cough (defined here as unexplained $\mathrm{CC}$ [UCC]) [13]. Particularly for investigative clinical trials, it is important to confirm a patient has RCC/ UCC to reliably evaluate novel treatments. The mechanisms underlying $\mathrm{CC}$ are under active investigation and are most likely heterogeneous [16]. However, many patients with $\mathrm{CC}$ share a common history of a cough triggered by low levels of thermal, mechanical, or chemical stimuli, including innocuous stimuli (allotussia), a sensation of itching or tickling in the throat (laryngeal paresthesia) accompanied by an urge to cough, and increased responsiveness to tussive stimuli (hypertussia) [17]. Cough hypersensitivity syndrome has been suggested as a useful clinical paradigm to describe these patients [13, 16-18].

An understanding of the typical profile of patients with $\mathrm{CC}$ is important to recognize the unique features of such patients. Epidemiologic studies have revealed typical features of patients with $\mathrm{CC}$, including a preponderance of females who have never smoked $[4,11,19-21]$. The prevalence of CC peaks around middle age (i.e., in the fifth and sixth decades), though CC can occur in all age groups [22]. Patients with CC can be evaluated by using objective and subjective methods that measure different aspects of cough, including objective cough frequency, cough severity (e.g., with the Cough Severity Diary [CSD] or cough severity visual analog scale [VAS]), and cough-specific health-related quality of life (e.g., with the Leicester Cough Questionnaire [LCQ] or Cough-Specific Quality-of-Life Questionnaire) [23]. Although objective cough monitoring is used in clinical trials, patient-reported measures, such as a simple cough score (e.g., asking patients to score their cough severity on a scale from 1 to 10 ), are more widely used in clinical practice [10].

Most prior observational studies of CC involved single clinics and predominantly included patients with explained $\mathrm{CC}$, with only a small proportion of RCC or UCC cases. The objective of this analysis was to characterize patients with RCC or UCC who were enrolled in a multicenter phase 2 study using a comprehensive, protocol-driven data collection approach to better define this patient population.

\section{Methods}

\section{Study Design and Patient Population}

This analysis assessed baseline demographics and characteristics of patients enrolled in a phase $2 \mathrm{~b}$, randomized, placebo-controlled trial of the P2X3-receptor antagonist gefapixant (ClinicalTrials.gov identifier, NCT02612610). Details regarding study design have been published [24]. For the current analysis, patients receiving any gefapixant dose or placebo were pooled into a single group for assessment of baseline characteristics.

Eligible patients were defined in the study protocol as having RCC or UCC based on American College of Chest Physicians (ACCP) and British Thoracic Society (BTS) guidelines. Patients were considered to have RCC if they had a clinical evaluation that identified at least one comorbid condition associated with $\mathrm{CC}$ but continued to cough despite receiving appropriate diagnostic workup and at least 2 months of therapy for the comorbid condition(s). Patients were defined as having UCC if there was no objective evidence of a comorbid condition associated with $\mathrm{CC}$ despite appropriate diagnostic workup per ACCP and BTS guidelines. Patients were required to have a minimum RCC or UCC duration of 1 year.

Additional inclusion criteria were age limits from 18 to 80 years and cough severity score of $\geq 40 \mathrm{~mm}$ on a $100-\mathrm{mm}$ cough severity VAS at screening. Exclusion criteria included current smoking or recent smoking within 6 months of enrollment, a ratio of forced expiratory volume in 1 second to forced vital capacity of less than $60 \%$, treatment initiation with an ACE inhibitor within 4 weeks of or during the study, use of opioids within 1 week of the study, or an upper or lower respiratory tract infection within 4 weeks of the study.

The study was performed in accordance with the International Council for Harmonisation-E6 Guideline for Good 
Clinical Practice and applicable federal regulations. All patients provided written informed consent before enrollment and all sites received approval from institutional review boards or independent ethics committees.

\section{Analysis Measures}

Baseline cough-related characteristics included objective awake cough frequency and patient-reported outcomes. Objective cough frequency was measured at baseline in a 24-hours sound recording using a VitaloJAK (Vitalograph ${ }^{\circledR}$; Vitalograph Ltd, Buckingham, United Kingdom) acoustic recording device as previously described $[24,25]$. Patientreported cough severity was scored from 0 to 100 using a 100-mm cough severity VAS (recall period: "past 24 hours"; $0=$ not at all; $100=$ extremely). Cough frequency, intensity, and disruption were assessed via the CSD, a 7-item questionnaire designed to be answered before bedtime (recall period: "today"); each item was measured on an 11-point numeric rating scale ranging from 0 (best) to 10 (worst). The CSD total score (range: 0 to 10 ) was calculated by averaging scores from all 7 items. Individual domain scores were calculated as the mean across items within each domain. The mean weekly CSD total score was calculated as the average CSD total score over the preceding week. Physical, psychological, and social burdens of cough were assessed using the LCQ, a 19-item health-related quality-of-life questionnaire (recall period: "last 2 weeks"). Each LCQ item was measured on a 7-point Likert scale, with lower numbers reflecting more severe cough effects. Individual scores for physical, psychological, and social domains were calculated as the average score across individual items within the domain; the LCQ total score (range: 3 to 21) was calculated as the sum of individual domain scores. The LCQ and CSD have been previously demonstrated to be reliable, responsive, and valid in patients with CC [26, 27]. Patients also completed a medical history questionnaire, developed for the purposes of this analysis, which comprised 21 items regarding various cough triggers. Responses regarding experience of individual triggers were categorized as either no (occurring "never" or "little of the time") or yes (occurring "some of the time," "a lot of the time," "most of the time," or "always").

\section{Data Collection and Analysis}

Medical history, including CC history, medication history (including over-the-counter medications administered 30 days before screening and $\mathrm{CC}$ treatments within 1 year before screening), and patient demographics were collected during screening. Comorbid conditions were identified on the basis of medical history in patient medical records. Medical history terms were coded using the MedDRA v19.0.
At the baseline visit, inclusion and exclusion criteria were confirmed, an updated medical history was obtained, concomitant medications were recorded, and baseline cough metrics (i.e., awake cough frequency, average of the daily CSD scores over the prior 7 days, cough severity VAS, and LCQ) were collected. Data were summarized using descriptive statistics.

\section{Results}

\section{Patient Demographics and Characteristics}

A total of 253 patients met eligibility criteria and were enrolled in the study. Most patients were female (76\%), white (92\%), and never smokers (70\%). Median (range) age was 61 (22-79) years. On average, patients were overweight (mean body mass index, $27.7 \mathrm{~kg} / \mathrm{m}^{2}$ ). All enrolled patients were from the United States or United Kingdom (Table 1).

\section{Baseline Cough Characteristics}

Patients enrolled in this study had severe and long-lasting coughs (Table 2). Although eligibility criteria required patients to have a cough lasting for at least 1 year, the median duration of cough was much longer (11.0 years). Median awake cough frequency was 28.9 coughs per hour but ranged from less than 1 to more than 700 coughs per hour. Consistent with eligibility criteria, patients reported a mean baseline cough severity VAS score of $57.5 \mathrm{~mm}$.

The mean total LCQ score at baseline was 11.7, with mean scores of 4.4, 3.7, and 3.5 for the physical, psychological, and social domains, respectively. Baseline total LCQ scores ranged from 4.2 to 19.7. Individual LCQ items with the highest reported burden (i.e., lowest scores) included psychological factors (did not feel in control of cough, embarrassment, frustration) and social factors (annoying to partner, family, and friends; interrupted conversations). Items in the physical domain were typically reported as less burdensome compared with the psychological and social domains, with chest/stomach pains and hoarse voice among the items with lowest reported overall burden (Fig. 1).

The mean weekly CSD total score at baseline was 4.2, with mean scores of 4.9, 4.4, and 2.9 for the frequency, intensity, and disruption domains. Baseline mean weekly CSD total scores ranged from 0.8 to 9.9 . Items that were rated as most burdensome were in the frequency (frequency of urge to cough, cough frequency) and intensity (harshness of cough) domains (Fig. 2). Items related to disruption (disruption of activities or sleep) were rated as least burdensome. 
Table 1 Baseline demographics and clinical characteristics [24]

\begin{tabular}{|c|c|}
\hline Parameter & $\begin{array}{l}\text { Patient popula- } \\
\text { tion }(N=253)\end{array}$ \\
\hline \multicolumn{2}{|l|}{ Sex, $n(\%)$} \\
\hline Female & $193(76)$ \\
\hline Male & $60(24)$ \\
\hline \multicolumn{2}{|l|}{ Ethnicity, $n(\%)$} \\
\hline Hispanic or Latino & $3(1)$ \\
\hline Not Hispanic or Latino & 250 (99) \\
\hline \multicolumn{2}{|l|}{ Race, $n(\%)$} \\
\hline American Indian or Alaskan native & $2(1)$ \\
\hline Asian & $3(1)$ \\
\hline Black or African American & $12(5)$ \\
\hline White & $234(92)$ \\
\hline Other & $1(<1)$ \\
\hline Multiple & $1(<1)$ \\
\hline \multicolumn{2}{|l|}{ Country, $n(\%)$} \\
\hline United Kingdom & $88(35)$ \\
\hline United States & $165(65)$ \\
\hline \multicolumn{2}{|l|}{ Age, years } \\
\hline Mean (SD) & $60(10)$ \\
\hline Median (range) & $61(22-79)$ \\
\hline \multicolumn{2}{|l|}{ Smoking status, $n(\%)$} \\
\hline Never & $177(70)$ \\
\hline Former & $76(30)$ \\
\hline \multicolumn{2}{|l|}{ BMI, $\mathrm{kg} / \mathrm{m}^{2}$} \\
\hline Mean (SD) & $27.7(4.7)$ \\
\hline \multicolumn{2}{|l|}{$\mathrm{FEV}_{1} / \mathrm{FVC}, \%$} \\
\hline Mean (SD) & $81.7(12.2)$ \\
\hline
\end{tabular}

$B M I$ body mass index, $F E V_{1}$ forced expiratory volume in 1 second, $F V C$ forced vital capacity, $S D$ standard deviation

Each cough trigger included in the baseline 21-item questionnaire was self-reported to elicit cough at least some of the time by more than $30 \%$ of patients (Table 3 ). The most common triggers of cough were irritation in the throat $(88 \%)$ and a tickle in the throat $(85 \%)$. Only $4 \%$ to $5 \%$ of patients reported that irritation or tickling in the throat never elicited their cough. The most common external triggers were poor air quality $(74 \%)$ and change in air temperature (71\%). The least commonly reported triggers were crying (32\%), swallowing (35\%), and certain foods (44\%). Nearly two-thirds of patients $(65 \%)$ reported there were no specific triggers for their cough and $90 \%$ reported their coughing was often unpredictable.
Table 2 Baseline cough characteristics

\begin{tabular}{ll}
\hline Parameter & Patient population $(N=253)$ \\
\hline Cough duration, years & \\
Mean (SD) & $14.2(11.1)$ \\
Median (range) & $11.0(2.0,56.0)$ \\
Awake cough frequency, coughs/hour ${ }^{\mathrm{a}}$ & \\
Mean (SD) & $40.3(55.8)$ \\
Geometric mean & 26.9 \\
Median (range) & $28.9(0.4,734.0)$ \\
LCQ total score & $11.7(3.0)$ \\
Mean (SD) & $11.7(4.2,19.7)$ \\
Median (range) & \\
Cough severity VAS score, mm ${ }^{\mathrm{b}}$ & $57.5(22.3)$ \\
Mean (SD) & $60.0(7.0,100.0)$ \\
Median (range) & \\
Daily CSD total score & \\
Mean (SD) & $4.2(1.9)$ \\
Median (range) & $4.1(0.8,9.9)$ \\
\hline
\end{tabular}

$C S D$ cough severity diary, $L C Q$ Leicester Cough Questionnaire, $S D$ standard deviation, $V A S$ visual analog scale

${ }^{a}$ Includes patients who received at least one dose of placebo or gefapixant and provided one or more baseline and one or more postbaseline measures of awake cough frequency $(N=236)$

${ }^{\mathrm{b}}$ Baseline cough severity VAS scores were collected 1 to 14 days after collection of cough severity VAS scores to determine trial eligibility during screening

${ }^{\mathrm{c}} N=246$ for baseline CSD

\section{Medical History and Comorbidities}

Common comorbidities associated with $\mathrm{CC}$ were prevalent in the patient population; $56 \%, 30 \%$, and $47 \%$ of patients had a diagnosis of GERD, asthma, and allergic rhinitis, respectively, recorded in their medical history (Fig. 3a). Overall, 29\% of patients had a single diagnosis of one of these conditions (Fig. 3b), 33\% had dual diagnoses, and $12 \%$ had diagnoses of all 3 conditions (Fig. 3c).

The most common classes of prior or comorbid conditions were identified using patient medical records (Table 4). Several individual-condition medical history items were consistent with complications or comorbidities related to cough, including headache $(15 \%)$, hiatus hernia $(13 \%)$, stress urinary incontinence $(5 \%)$, and bronchitis $(4 \%)$.

The most common medication classes were consistent with cough treatments and associated comorbid conditions (Table 5). The most common individual medications used before enrollment that were presumed to be cough related included gabapentin (11\%), benzonatate $(9 \%)$, omeprazole $(8 \%)$, prednisone $(7 \%)$, bromhexine $(6 \%)$, azelastine hydrochloride $(6 \%)$, and guaifenesin $(5 \%)$. 


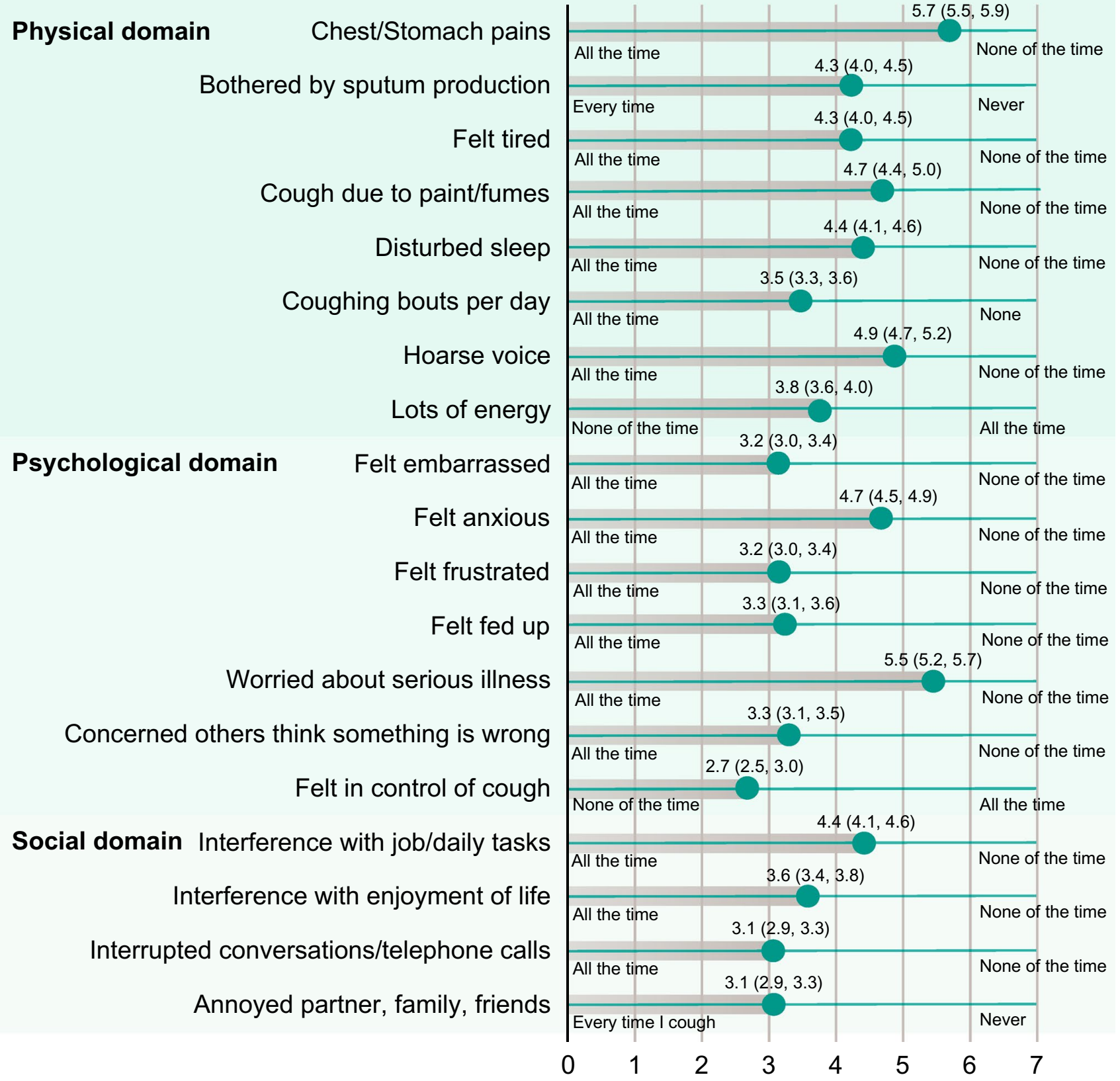

Fig. 1 Individual items in the Leicester Cough Questionnaire reported by patients at baseline. Data expressed in mean (95\% confidence interval)

\section{Conclusion}

This analysis assessed characteristics of patients with RCC/ UCC enrolled in a large phase 2 study. Patients were typically female, middle aged, never smokers, and overweight. They presented with long-lasting, severe, and burdensome cough, as evidenced by a median cough duration of more than a decade, high awake cough frequency, and severe cough based on patient-reported outcomes (i.e., CSD, cough severity VAS). Patients also had an impaired cough-specific health-related quality of life, with the highest self-reported burden being related to social and psychological impairment. Patients reported a wide range of cough triggers, including those implicated in cough hypersensitivity syndrome (e.g., $95 \%$ to $96 \%$ of patients reported at least some throat tickle or irritation). Patients frequently had 1 or more diagnoses of conditions thought to be associated with $\mathrm{CC}$.

The clinical profile of patients with RCC/UCC enrolled in this phase 2 study is generally consistent with that of several previous epidemiologic and observational CC studies, although they included patients with explained $\mathrm{CC}$ and did not select by cough severity [4, 20-22]. Particularly, a 


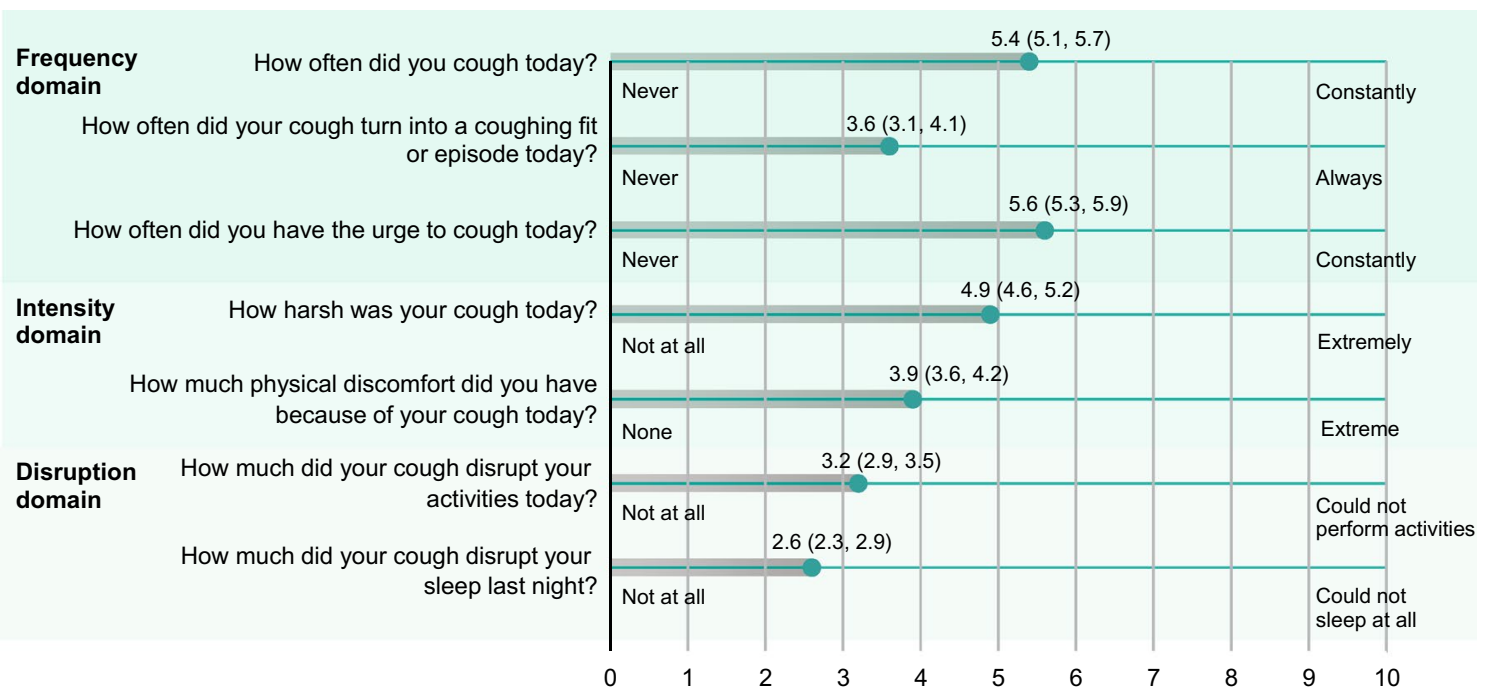

Fig. 2 Individual items in the Cough Severity Diary reported by patients at baseline. Data expressed in mean (95\% confidence interval)

Table 3 Reported triggers of cough $(n=252)$

\begin{tabular}{llc}
\hline Item & $\begin{array}{l}\text { Yes (some of the time to } \\
\text { always) } n(\%)\end{array}$ & $\begin{array}{l}\text { No (little of the } \\
\text { time to } \text { never } n \\
(\%)\end{array}$ \\
\hline My cough is unpredictable & $228(90)$ & $24(10)$ \\
An irritation in my throat triggers my cough & $223(88)$ & $29(12)$ \\
A tickle in my throat triggers my cough & $213(85)$ & $38(15)$ \\
Poor air quality triggers my cough & $187(74)$ & $65(26)$ \\
A change in air temperature triggers my cough & $180(71)$ & $72(29)$ \\
I cough because I need to clear my throat & $180(71)$ & $72(29)$ \\
Deep breathing makes me cough & $165(65)$ & $87(35)$ \\
There are no specific triggers for my cough & $163(65)$ & $89(35)$ \\
Exertion or exercise makes me cough & $163(65)$ & $89(35)$ \\
A dry throat triggers my cough & $158(63)$ & $94(37)$ \\
Laughing triggers my cough & $155(62)$ & $97(38)$ \\
I cough after meals & $148(59)$ & $104(41)$ \\
Lying flat makes me cough & $148(59)$ & $104(41)$ \\
Changes in the weather trigger my cough & $144(57)$ & $108(43)$ \\
A high pollen count triggers my cough & $142(56)$ & $110(44)$ \\
Breathlessness causes me to cough & $142(56)$ & $110(44)$ \\
My cough is triggered by certain smells and odors & $140(56)$ & $112(44)$ \\
An irritation in my chest has triggered my cough & $128(51)$ & $123(49)$ \\
My cough is triggered by certain foods & $111(44)$ & $141(56)$ \\
Swallowing triggers my cough & $89(35)$ & $163(65)$ \\
Crying triggers my cough & $80(32)$ & $172(68)$
\end{tabular}

One patient did not complete the trigger questionnaire at baseline retrospective study of 10,032 patients with CC who attended specialist cough clinics and did not have significant radiologic abnormalities found that patients were predominantly female (66\%), had a mean age of 55 years, and had a common age of presentation in the sixth decade, consistent with demographics observed in the current analysis [22]. However, the proportion of female patients in the current study $(76 \%)$ was notably greater than the proportion of females $(57 \%)$ in a previous large population-based study that included 554 individuals with CC [21]. This analysis 
a

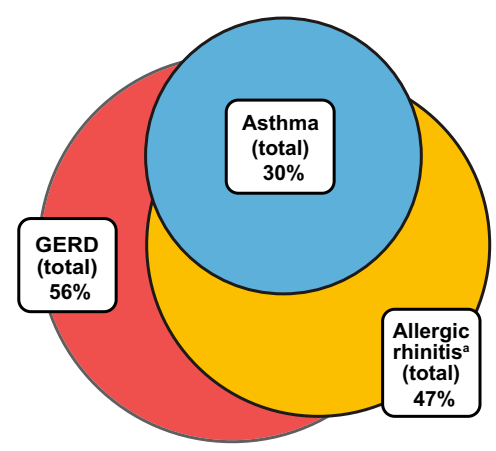

b

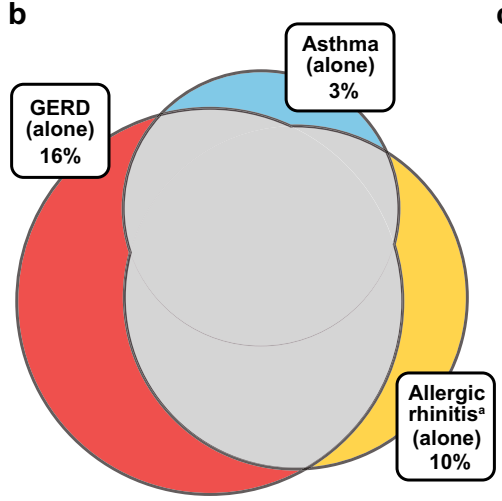

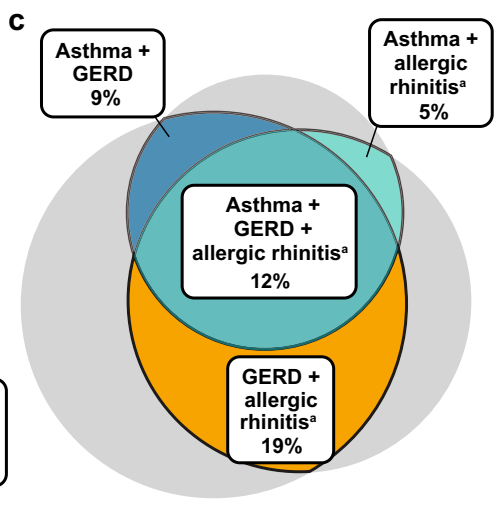

Fig. 3 Most common medical conditions associated with chronic cough in the patient population. a Percentage of patients with a medical history of GERD, asthma, or allergic rhinitis; b percentage of patients with a single diagnosis of GERD, asthma, or allergic rhinitis; and $\mathbf{c}$ percentage of patients with dual or all 3 diagnoses of GERD, asthma, or allergic rhinitis. GERD, gastroesophageal reflux disease. ${ }^{a}$ Diagnostic codes in allergic rhinitis category include seasonal rhinitis, seasonal allergic rhinitis, seasonal allergies rhinitis, perennial seasonal allergic rhinitis (dust mite), and seasonal allergic rhinitis (trees weeds grass)
Table 4 Commonly reported medical conditions by body system class $(>20 \%)$

\begin{tabular}{lc}
\hline Parameter & $\begin{array}{l}\text { Patient popula- } \\
\text { tion }(n=252) \\
n(\%)\end{array}$ \\
\hline Respiratory, thoracic, and mediastinal disorders (including cough) & $252(100)$ \\
Surgical and medical procedures & $181(72)$ \\
Gastrointestinal disorders (e.g., gastroesophageal reflux disease) & $174(69)$ \\
Musculoskeletal and connective tissue disorders & $139(55)$ \\
Nervous system disorders & $121(48)$ \\
Infections and infestations & $112(44)$ \\
Social circumstances (e.g., menopause, postmenopause) & $106(42)$ \\
Metabolism and nutrition disorders & $99(39)$ \\
Vascular disorders & $97(38)$ \\
Psychiatric disorders & $92(37)$ \\
Immune system disorders & $84(33)$ \\
Skin and subcutaneous tissue disorders & $56(22)$
\end{tabular}

One patient did not report medical history
Table 5 Most common prior therapies $(n=252)$

\begin{tabular}{ll}
\hline Medication class & $n(\%)$ \\
\hline Steroids (inhaled or oral) & $54(21)$ \\
Antihistamines $_{\text {Stomach acid reducers }}{ }^{\mathrm{a}}$ & $39(15)$ \\
Bronchodilators & $39(15)$ \\
Benzonatate & $36(14)$ \\
Opioids & $23(9)$ \\
\hline
\end{tabular}

Includes all medications, including over-the-counter medications, over the past 30 days that were recorded, in addition to all chronic cough treatments over the past year at screening

One patient did not report medical history

${ }^{a}$ Includes proton pump inhibitors, antacids, and $\mathrm{H}_{2}$-receptor antagonists expands upon previous studies by providing additional, prospective characterization of the RCC/UCC patient population using both objective and subjective measures. Although the clinical profile identified in this and previous studies may help identify these patients in a clinical setting, a definitive diagnosis of RCC/UCC should be made using established guidelines $[1,10]$. Of note, the current study was not designed to compare characteristics between patients diagnosed with RCC vs UCC, and future research comparing these populations may be warranted to assess whether these diagnostic labels reflect distinct patient populations.

Strengths of the analysis include a large sample size of a well-defined group of patients with RCC or UCC. The use of a clinical study protocol with defined eligibility criteria is more likely to include a consistent diagnosis of RCC/UCC 
than that reported in observational studies. Additionally, the study protocol facilitated collection of information that may not be readily available in observational studies, including medical history, prior treatments and medications, awake cough frequency, and patient-reported outcomes.

There were limitations to this analysis. First, the selected clinical study population of patients with RCC/UCC with a cough severity VAS score of $>40 \mathrm{~mm}$ may not represent patients with less severe RCC/UCC. Second, medical histories, including comorbid conditions, were identified from diagnoses recorded in patients' medical records; although clinical diagnoses (such as GERD and UACS) may have been accurately captured, medical conditions that relied on patient reports could have been underestimated because specific questions were not asked. For example, the prevalence of stress urinary incontinence observed in this study (5\%) was much lower than the prevalence of stress urinary incontinence reported in a study that used targeted questions to estimate the prevalence of incontinence in women with CC (63\%) [28]. Third, prior medications and CC treatments were only recorded over the past 30 days and 1 year, respectively; thus, only current or recent treatments were captured. Of note, patients enrolled in this study had experienced CC for many years, so collection of a comprehensive medication history would be challenging. Fourth, the medical history questionnaire used to collect data regarding cough triggers did not have a defined recall period and was not intended to capture potential variation in cough-trigger prevalence over time. Finally, all enrolled patients were from the United States or United Kingdom; it is therefore unclear how these results may generalize to patients from other geographic regions.

This analysis suggests that patients with RCC/UCC have a common clinical profile that is generally consistent with previous studies of patients with CC.

Acknowledgements This study was supported by Merck Sharp \& Dohme Corp., a subsidiary of Merck \& Co., Inc., Kenilworth, NJ, USA. Writing and editorial assistance were provided under the direction of the authors by Nathan Rodeberg, PhD, and Jenna Lewis, MA, ELS, of MedThink SciCom, with support from Merck Sharp \& Dohme Corp., a subsidiary of Merck \& Co., Inc., Kenilworth, NJ, USA.

Author Contributions All authors contributed to the conception of the analyses performed in this manuscript and interpretation of the data, provided critical review and revisions of the manuscript for intellectual content, and provided final approval for this manuscript.

Funding The clinical study was funded by Afferent Pharmaceuticals (acquired by Merck \& Co., Inc., Kenilworth, NJ, USA). The analysis and manuscript writing/editorial assistance were funded by Merck Sharp \& Dohme Corp., a subsidiary of Merck \& Co., Inc., Kenilworth, NJ, USA.

Data Availability The data sharing policy of Merck Sharp \& Dohme Corp., a subsidiary of Merck \& Co., Inc., Kenilworth, NJ, USA, including restrictions, is available at http://engagezone.msd.com/ds_ documentation.php. Requests for access to the clinical study data can be submitted through the EngageZone site or via email to dataaccess@ merck.com.

\section{Declarations}

Conflict of interest AHM has received consulting fees from Bayer, Bellus, Boehringer Ingelheim, Merck, Pfizer, Proctor \& Gamble, and Shionogi; lecture fees from AstraZeneca and Boehringer Ingelheim; and grant support from Afferent, Infirst, Merck, and Proctor \& Gamble. SSB has received grants from Merck \& Co., Inc.; scientific advisory board and consultancy fees from Bayer, Menlo, Merck \& Co., Inc., Patara, Pfizer, and Sanofi; speaker fees from Roche; and grants for travel and subsistence for attendance to scientific meetings from Boehringer Ingelheim. JAS has received grants and personal fees related to the submitted work from Afferent Pharmaceuticals/Merck \& Co., Inc.; grants from Ario Pharma, Bayer, Bellus, GlaxoSmithKline, Menlo, and NeRRe Pharmaceuticals; personal fees from Ario Pharma, Bayer, Bellus, Boehringer Ingleheim, Chiesi, GlaxoSmithKline, Genentech, Menlo, Neomed, and NeRRe Pharmaceuticals; nonfinancial support from Vitalograph; and is a named inventor on a patent, owned by Manchester University NHS Foundation Trust and licensed to Vitalograph Ltd, describing the detection of cough from sound recordings. JAS is also funded by the NIHR Manchester Biomedical Research Centre and a Wellcome Investigator Award and is an NIHR Senior Investigator. LPM has received grants from Afferent Pharmaceuticals/ Merck \& Co., Inc., British Heart Foundation, Chiesi, EU Interreg VA Health \& Life Science Programme, and NC3Rs; personal fees from Afferent Pharmaceuticals/Merck \& Co., Inc., Applied Clinical Intelligence, and AstraZeneca; grants for travel and subsistence for attendance to scientific meetings from Boehringer Ingelheim, Chiesi, and GlaxoSmithKline; and advisory board/consultancy fees from Almirall, Boehringer Ingelheim, GlaxoSmithKline, and NAPP. JS, AMN, ZJX, W-CW, and DRM are employees of Merck Sharp \& Dohme Corp., a subsidiary of Merck \& Co., Inc., Kenilworth, NJ, USA. MRS has received grants and personal fees from Afferent Pharmaceuticals/Merck \& Co., Inc. and is a consultant for AstraZeneca, Bayer, Bellus, NeRRe Therapeutics, and Nocion.

Ethical Approval This study was performed in accordance with the ethical standards of the Declaration of Helsinki. Approval of the clinical study (NCT02612610) at each study site was granted by one of the following institutional review boards (IRBs)/independent ethics committees: the Health Research Authority NRES Committee (Jarrow, UK), Sterling IRB (Atlanta, GA), Western IRB (Puyallup, WA), University of Utah IRB (Salt Lake City, UT), Kaiser Permanente Southern California IRB (Pasadena, CA), St. Luke's Hospital IRB (Chesterfield, MO), or Mayo Clinic IRB (Rochester, MN).

Consent to Participate All patients provided written informed consent before enrollment.

Open Access This article is licensed under a Creative Commons Attribution 4.0 International License, which permits use, sharing, adaptation, distribution and reproduction in any medium or format, as long as you give appropriate credit to the original author(s) and the source, provide a link to the Creative Commons licence, and indicate if changes were made. The images or other third party material in this article are included in the article's Creative Commons licence, unless indicated otherwise in a credit line to the material. If material is not included in the article's Creative Commons licence and your intended use is not permitted by statutory regulation or exceeds the permitted use, you will 
need to obtain permission directly from the copyright holder. To view a copy of this licence, visit http://creativecommons.org/licenses/by/4.0/.

\section{References}

1. Irwin RS, French CL, Chang AB et al (2018) Classification of cough as a symptom in adults and management algorithms: CHEST Guideline and Expert Panel Report. Chest 153:196-209

2. Song W-J, Chang Y-S, Faruqi S et al (2015) The global epidemiology of chronic cough in adults: a systematic review and metaanalysis. Eur Respir J 45:1479-1481

3. Kuzniar TJ, Morgenthaler TI, Afessa B et al (2007) Chronic cough from the patient's perspective. Mayo Clin Proc 82:56-60

4. Chamberlain SAF, Garrod R, Douiri A et al (2015) The impact of chronic cough: a cross-sectional European survey. Lung 193:401-408

5. Everett CF, Kastelik JA, Thompson RH et al (2007) Chronic persistent cough in the community: a questionnaire survey. Cough 3:5

6. Yousaf N, Montinero W, Birring SS et al (2013) The long term outcome of patients with unexplained chronic cough. Respir Med 107:408-412

7. Koskela HO, Lätti AM, Purokivi MK (2017) Long-term prognosis of chronic cough: a prospective, observational cohort study. BMC Pulm Med 17:146

8. Kang S-Y, Song W-J, Won H-K et al (2020) Cough persistence in adults with chronic cough: a 4-year retrospective cohort study. Allergol Int 69:588-593

9. Good JT Jr, Rollins DR, Kolakowski CA et al (2018) New insights in the diagnosis of chronic refractory cough. Respir Med 141:103-110

10. Morice AH, Millqvist E, Bieksiene K et al (2020) ERS guidelines on the diagnosis and treatment of chronic cough in adults and children. Eur Respir J 55:1901136

11. Zeiger RS, Schatz M, Butler RK et al (2020) Burden of specialistdiagnosed chronic cough in adults. J Allergy Clin Immunol Pract 8:1645-1657

12. Koskela HO, Lätti AM, Pekkanen J (2019) Risk factors for repetitive doctor's consultations due to cough: a cross-sectional study in a Finnish employed population. BMJ Open 9:e030945

13. McGarvey L, Gibson PG (2019) What is chronic cough? Terminology. J Allergy Clin Immunol Pract 7:1711-1714

14. French CT, Diekemper RL, Irwin RS et al (2015) Assessment of intervention fidelity and recommendations for researchers conducting studies on the diagnosis and treatment of chronic cough in the adult: CHEST Guideline and Expert Panel Report. Chest 148:32-54
15. Song M-K, Happ MB, Sandelowski M (2010) Development of a tool to assess fidelity to a psycho-educational intervention. J Adv Nurs 66:673-682

16. Mazzone SB, Chung KF, McGarvey L (2018) The heterogeneity of chronic cough: a case for endotypes of cough hypersensitivity. Lancet Respir Med 6:636-646

17. Song WJ, Morice AH (2017) Cough hypersensitivity syndrome: a few more steps forward. Allergy Asthma Immunol Res 9:394-402

18. Morice AH, Millqvist E, Belvisi MG et al (2014) Expert opinion on the cough hypersensitivity syndrome in respiratory medicine. Eur Respir J 44:1132-1148

19. French CL, Crawford SL, Bova C et al (2017) Change in psychological, physiological, and situational factors in adults after treatment of chronic cough. Chest 152:547-562

20. Campi G, Noale M, Fabbrizzi A et al (2020) The demographic and clinical characteristics of an Italian population of adult outpatients with chronic cough. Aging Clin Exp Res 32:741-746

21. Çolak Y, Nordestgaard BG, Laursen LC et al (2017) Risk factors for chronic cough among 14,669 individuals from the general population. Chest 152:563-573

22. Morice AH, Jakes AD, Faruqi S et al (2014) A worldwide survey of chronic cough: a manifestation of enhanced somatosensory response. Eur Respir J 44:1149-1155

23. Birring SS, Spinou A (2015) How best to measure cough clinically. Curr Opin Pharmacol 22:37-40

24. Smith JA, Kitt MM, Morice AH et al (2020) Gefapixant, a P2X3 receptor antagonist, for the treatment of refractory or unexplained chronic cough: a randomised, double-blind, controlled, parallelgroup, phase $2 b$ trial. Lancet Respir Med 8:775-785

25. Kelsall A, Decalmer S, Webster D et al (2008) How to quantify coughing: correlations with quality of life in chronic cough. Eur Respir J 32:175-179

26. Martin Nguyen A, Bacci E, Dicpinigaitis P et al (2020) Quantitative measurement properties and score interpretation of the cough severity diary in patients with chronic cough. Ther Adv Respir Dis 14:1753466620915155

27. Birring SS, Prudon B, Carr AJ et al (2003) Development of a symptom specific health status measure for patients with chronic cough: Leicester Cough Questionnaire (LCQ). Thorax 58:339-343

28. Dicpinigaitis PV (2021) Prevalence of stress urinary incontinence in women presenting for evaluation of chronic cough. ERJ Open Res 7:00012-02021

Publisher's Note Springer Nature remains neutral with regard to jurisdictional claims in published maps and institutional affiliations. 